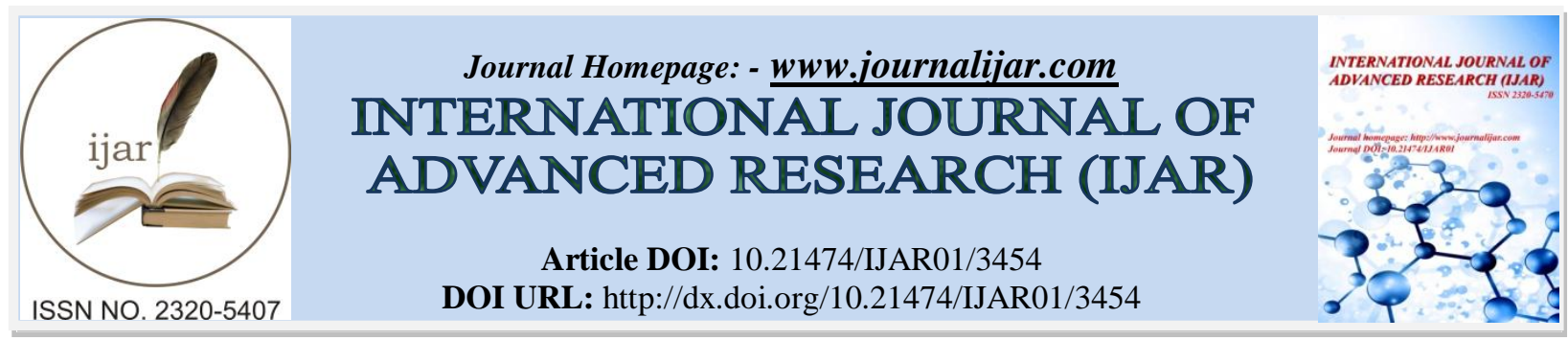

RESEARCH ARTICLE

\title{
TRACHEAL BRONCHUS AS AN INCIDENTAL FINDING IN TRAUMA PATIENT: A CASE REPORT
}

\author{
Kharaba Ayman MD ${ }^{1}$, Sherbini Nahid $\mathrm{MD}^{2}$,Al YamiKhaled $\mathrm{MS}^{3}$, KharabahReham $\mathrm{MS}^{3}$ and Albeihany \\ Amal MD'. \\ 1. Department of Critical Care, King Fahad hospital, Madinah, Saudi Arabia. \\ 2. Department Of Medicine, King Fahad hospital, Madinah, Saudi Arabia. \\ 3. Taibah University, Madinah, Saudi Arabia.
}

\section{Manuscript Info}

Manuscript History

Received: 13 December 2016

Final Accepted: 17 January 2017

Published: February 2017

\begin{abstract}
Congenital conditions of the major airways are very uncommon. Tracheal bronchus is the commonest,it affects $0.1-2 \%$ of the population. Usually asymptomatic but especially in childhood they may present with cough, recurrent infections, or hemoptysis. We describe a 25 -years-old male trauma patient with a right -sided tracheal bronchus arising from the lateral wall of the trachea (Conacher's carinal trifurcation TYPEIII) found incidentally on routine chest radiography upon admission to intensive care unit which confirmed by chest computed tomography and bronchoscopy. In adults with tracheal bronchus, the main concerns are complications during endotracheal intubation, risk of obstructive pneumonia, prolong hypoxia, lung neoplasms and the association with cardiovascular, renal and other congenital anomalies.
\end{abstract}

Copy Right, IJAR, 2017,. All rights reserved.

\section{Introduction:-}

Major airways congenital conditions are very uncommon, the most common anomaly is a tracheal bronchus that rises from the lateral wall of the trachea above the carina and supplies the right upper lobe ,the prevalence of tracheal bronchus reported is $0.1-2 \%$ while the incidence was found to be $0.06 \%$ in other evidence $(1,2)$. This anomaly is usually asymptomatic, but it may present with respiratory manifestations especially in childhood (3). It is also referred to as "bronchus suis" or "pig's bronchus" because it is a normal anatomical finding in pigs,sheep, goats, and camels.

In intensive care units, intubation and bronchoscopy are done frequently for a variety of causes. However, the anatomical variants of the airways are not routinely included in the differential diagnosis either for consideration of possible endotracheal intubation complications, a recurrent infection, persistent cough, or hemoptysis, which may be associated with existence of pulmonary orcardiovascular anomalies or lung cancer in these cases $(4,5)$. Here, we report a case of tracheal bronchus detected incidentally in the intensive care unit during a daily practice.

\section{Case Report:-}

A 25-year-old manwas admitted to the intensive care unit with multiple traumatic head injuries sustained in a motorcycle accident. He was intubated and placed on mechanical ventilation to protect his airway and remained in a coma in the intensive care unit for one month. 
He had no history of respiratory symptoms or admissions for any other indications. A review of systems was unremarkable, and physical examination revealed no clubbing of fingers and normal respiratory and cardiac examination. One pupil was dilated, and his score on the Glasgow coma scale was 7/15.

Chest radiographs ( $\mathrm{X}$ rays and computed tomography), taken as part of complete trauma assessment at presentation (Figure 1) revealed a tracheal bronchus originating from the right lateral wall of the trachea, just above the carina, and supplying the complete right upper lobe.

After 10 days in the intensive care unit, he underwent tracheostomy with a flexible bronchoscope (Figure 2). During the procedure, we confirmed the finding of three openings: a tracheal bronchus to the right upper lobe with three clear segments, an opening from the carina supplying the right bronchus intermedius and the right lower lobe segments, and an opening from the carina supplying the left lobes. All openings originated at the level of the carina .

Figure 1:- Tracheal bronchus originating from the right lateral wall of the trachea, just above the carina, supplying the complete right upper lobe in a 25 -year-old man admitted to the intensive care unit with multiple traumatic head

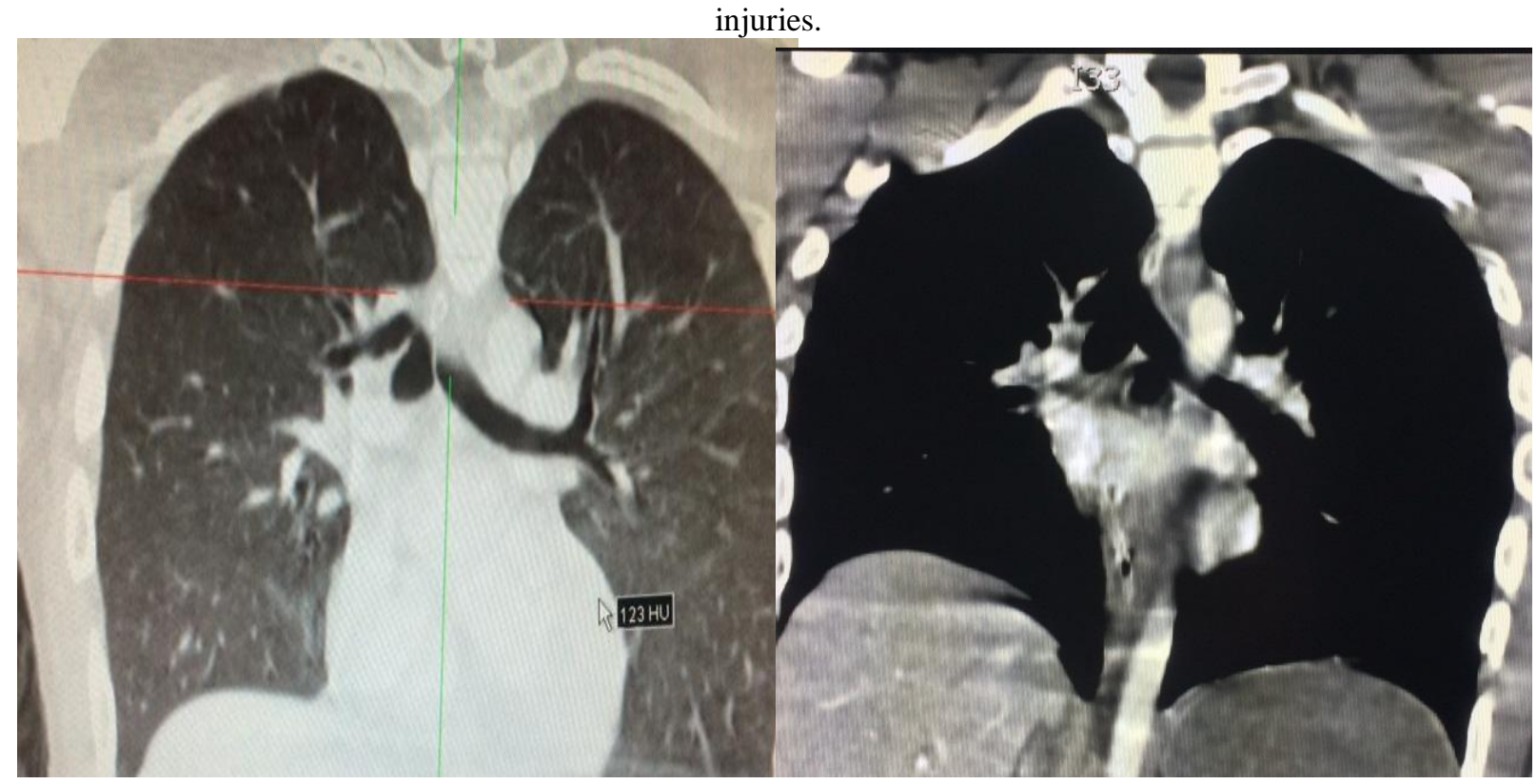

Figure 2:- Bronchoscopy revealed that the tracheal bronchus originated in the lateral wall of the trachea, less than 2 $\mathrm{cm}$ above the carina (Conacher's carinal trifurcation TYPE III).

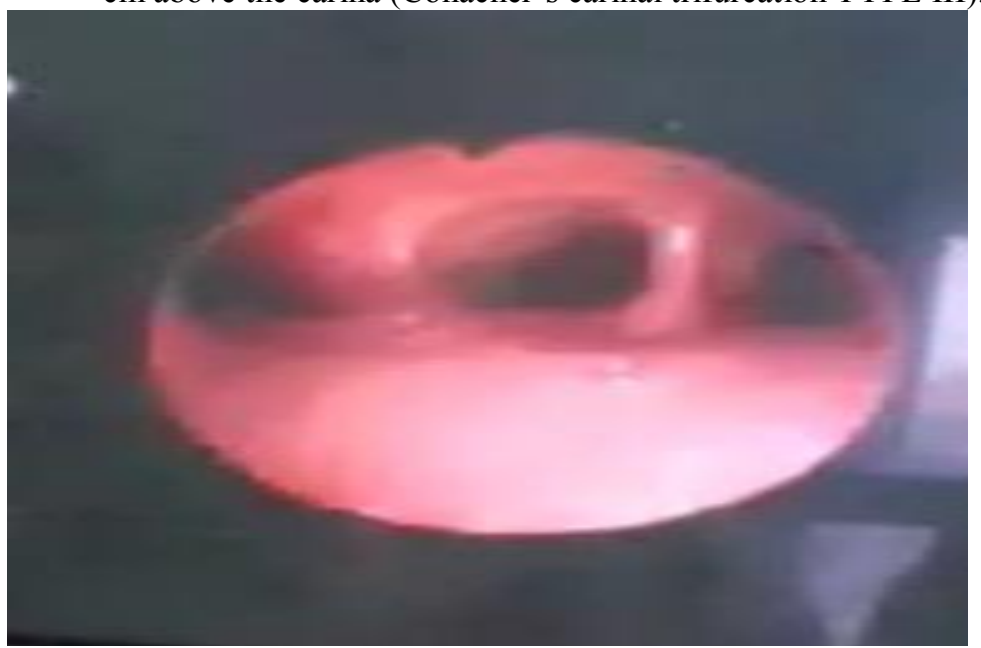




\section{Discussion:-}

Tracheal bronchus was first described by Sandifort in 1785 as a right upper-lobe bronchus or "true tracheal bronchus, "originating from the trachea. The term "tracheal bronchus" has come to include all bronchial anomalies originating from the trachea or main bronchus and directed to either the upper lobe of either the right or left lung. They classified into two main types to eithersupranumerary(bronchial supply to affected lung segment is present) or displaced (bronchial supply to affected lung segment is absent) (3).

These congenital malformations are usually asymptomatic in adults, but they are often associated with respiratory complications, recurrent infection, cough, wheeze, stridor, respiratory distress, and thoracic masses in children (6). Chest radiography, as the initial radiological investigation, may show a bronchus directly arising from the trachea, as it did in our case. Computed tomography is the imaging modality of choice for investigating this anomaly because it shows the tracheal and bronchial anatomy. This anomaly usually is detected incidentally during bronchoscopy or by chest computed tomography performed for various respiratory conditions $(6,7)$.

Occasionally, tracheal bronchus represents the underlying cause of a chronic pulmonary disease, such as emphysema, bronchiectasis or persistent or recurrent pneumonia, especially if it involves the right upper lobe and reflects difficulties in clearing mucus from this lobe $(3,4)$.

Tracheal bronchus may be associated with many anomalies including ventricular septal defect, atrial septal defect, pulmonary stenosis, coarctation of aorta, azygos lobe, tracheal stenosis, pulmonary hypoplasia, hydronephrosis, kidney agenesis, deafness and Down's syndrome (6-8).

Among various classifications, the one proposed by Conacher is simplest and describes the anatomical relationship between the tracheal bronchus and the carina. Types II and III may present a problem for Intensivist and anesthetists during intubation, especially if one lung needs to be ventilated (9).

In adults with a tracheal bronchus, presented in a critical condition to emergency department, intensive care unit or going for surgery the main concern is endotracheal intubation. An endotracheal tube can obstruct the lumen of the tracheal bronchus, resulting in atelectasis of the involved lobe, post-obstructive pneumonia, or even respiratory failure (10). Accidental intubations of the anomalous lobe can cause pneumothorax and inadequate ventilation of the remaining lung. Patients withtracheal bronchus should be identified and those aware to have this anomaly must inform the anesthesiologist preoperatively for those going forelective surgeryespecially cardiothoracic surgery with single-lung ventilation because serious prolong hypoxia and atelectasis may occur. (11-13).

In the absence of symptoms, tracheal bronchus need not be treated as in our patient where it went undiagnosed until his accident. In patients with a tracheal bronchus and right upper lobe disease with history ofrecurrent pneumonia which complicated by bronchiectasis, surgical resection of the anomalous lobe or segment is the treatment of choice (12-14).

\section{Conclusion:-}

Our patient was an adult with asymptomatic bronchial abnormalities with early identification for his anomaly at presentation, these tracheal anomaliesis important to be considered in daily practice to prevent endotracheal intubation complications and to identify the patients with chronic respiratory manifestations and for whom lobectomy may be considered. If a tracheal bronchus is detected, other pulmonary, cardiovascular and renal abnormalities should be ruled out. 


\section{References:-}

1. Ghaye B, Szapiro D, Fanchamps JM et-al. Congenital bronchial abnormalities revisited. Radiographics. 21 (1): 105-19. Radiographics

2. Findik S. Tracheal bronchus in the adult population. J BronchologyIntervPulmonol. 2011 Apr;18(2):149-53. doi: 10.1097/LBR.0b013e318216e30e.

3. Manjunatha YC, Gupta AK. Tracheal bronchus (pig bronchus). Indian J Pediatr. 2010 Sep;77(9):1037-8. doi: 10.1007/s12098-010-0162-6. Epub 2010 Aug 26.

4. Kumagae Y, Jinguji M, Tanaka D, Nakajo M. An adult case of bilateral true tracheal bronchi associated with hemoptysis. J Thorac Imaging. 2006 Nov;21(4):293-5.

5. Sezen CB, Celik A, Fazlioglu M, Memis L, Tastepe AI. Lung cancer developing from tracheal bronchus. Asian CardiovascThorac Ann. 2014 Jun 19. pii: 0218492314541450

6. Yong Seok Kim, MD,1 Tae Min Um, MD,1 Seung Kook Son, MD,1 Hye Young Kim, MD,1 Yong-Woo Kim, MD,2 and HeeJu Park, MD1.The Clinical Consideration of Tracheal Bronchus Detected by Computed Tomography Scan in Children. Pediatr Allergy Respir Dis. 2012 Dec; 22(4):411-416. Korean.http://dx.doi.org/10.7581/pard.2012.22.4.411

7. Shahul HA, Manu MK, Mohapatra AK. A rare case of coexisting left pulmonary hypoplasia and right trachealbronchus.BMJ Case Rep. 2014 Aug 25;2014. pii: bcr2014205008. doi: 10.1136/bcr-2014-205008.

8. Ghaye B, Szapiro D, Fanchamps JM, Dondelinger RF. Congenital bronchial abnormalities revisited. Radiographics. 2001;21(1):105-19.

9. Conacher ID. Implications of a tracheal bronchus for adult anaesthetic practice. Br J Anaesth. 2001;86:155-6.

10. Owen RL, Cheney FW. Endotracheal intubation: a preventable complication. Anesthesiology. 1987;67(2):2557.

11. Ikeno S, Mitsuhata H, Saito K, Hirabayashi Y, Akazawa S, Kasuda H, et al. Airway management for patients with a tracheal bronchus. Br J Anaesth. 1996;76(4):573-5.

12. Srivastava A, Warrier G, Trehan M, Radha AS. Tracheal bronchus: a cause of unexplained prolonged hypoxemia during ventilation. PaedtrCardiol. 2010;31(8):1229-31. http://dx.doi.org/10.1007/s00246- 010-97599.

13. Iwamoto T, Takasugi Y, Hiramatsu K, Koga Y, Konishi T, Kozuka K, et al. Three-dimensional CT image analysis of a tracheal bronchus in a patient undergoing cardiac surgery with one-lung ventilation. J Anesth. 2009;23(2):260-5.

14. Doolittle AM, Mair EA. Tracheal bronchus: classification, endoscopic analysis, and airway management. Otolaryngol Head Neck Surg. 2002;126:240-3. 